\title{
Net reaction rate and neutrino emissivity for the Urca process in departure from chemical equilibrium
}

\author{
Wei-Hua Wang, ${ }^{1, *}$ Xi Huang, ${ }^{1,2,3}$ and Xiao-Ping Zheng ${ }^{1, \dagger}$ \\ ${ }^{1}$ Institute of Astrophysics, Central China Normal University, \\ Wuhan 430079, China \\ ${ }^{2}$ Key Laboratory of Quark and Lepton Physics (Ministry of Education), \\ Central China Normal University, Wuhan 430079, China \\ ${ }^{3}$ School of Electronic and Electrical Engineering, \\ Wuhan Textile University, Wuhan 430073, China
}

\begin{abstract}
We discuss the effect of compression on Urca shells in the ocean and crust of accreting neutron stars, especially in superbursting sources. We find that Urca shells may be deviated from chemical equilibrium in neutron stars which accrete at several tenths of the local Eddington accretion rate. The deviation depends on the energy threshold of the parent and daughter nuclei, the transition strength, the temperature, and the local accretion rate. In a typical crust model of accreting neutron stars, the chemical departures range from a few tenths of $k_{B} T$ to tens of $k_{B} T$ for various Urca pairs. If the Urca shell can exist in crusts of accreting neutron stars, compression may enhance the net neutrino cooling rate by a factor of about $1 \sim 2$ relative to the neutrino emissivity in chemical equilibrium. For some cases, such as Urca pairs with small energy thresholds and/or weak transition strength, the large chemical departure may result in net heating rather than cooling, although the released heat can be small. Strong Urca pairs in the deep crust are hard to be deviated even in neutron stars accreting at the local Eddington accretion rate.

PACS numbers: $23.40 .-\mathrm{s}, 26.30 .+\mathrm{k}, 26.60 .+\mathrm{c}, 97.10 . \mathrm{Cv}$
\end{abstract}

\section{INTRODUCTION}

Superbursts are rare, long lasting and energetic x-ray flares which originate from accreting neutron stars in low-mass $\mathrm{x}$ ray binaries (LMXBs) [1-3]. The flares rise in a few seconds and decay in a few hours in an exponential-like way, and the fluences can be as large as $10^{42} \mathrm{erg}$. These characteristics distinguish them from "ordinary" Type-I x-ray bursts, which are 1000 times shorter, less energetic, and more frequent. Besides, the local accretion rates of the superbursting sources are supposed to be $\dot{m}=(0.1-0.3) \dot{m}_{E d d}$ [4], where $\dot{m}_{E d d} \simeq 10^{5} \mathrm{~g} \mathrm{~cm}^{-2} \mathrm{~s}^{-1}$ is the local Eddington accretion rate.

The current superburst model proposes that continual accretion compresses the preexisting material deeper into the crust, raises the chemical potential of electrons, $\mu_{e}$, and induces nonequilibrium reactions if the element transformation is energetically favorable. An energy of heat of about $\approx 1-2 \mathrm{MeV}$ per accreted nucleon will be deposited through electron capture (EC), neutron emissions, and pycnonuclear fusion [5-8]. Part of this energy flows upward to heat the neutron star ocean, where the superbursts are supposed to be triggered by unstable burning of ${ }^{12} \mathrm{C}[3,9-13]$ in column depth $y_{\text {ign }} \approx(0.5-3) \times 10^{12} \mathrm{~g} / \mathrm{cm}^{-2}[14]$.

The high temperature dependence of carbon burning [15] makes the ignition sensitive to deep crustal heating; accordingly, superburst observations have the potential to constrain the combined study of crustal heating and neutrino cooling of the crust and core [16], and also to act as probes of nu-

\footnotetext{
*wangweihua@mails.ccnu.edu.cn

†zhxp@phy.ccnu.edu.cn
}

clear physics [14]. However, the actual crust temperature is set by deep crustal heating, neutrino cooling in the crust and core, and heat transport from the interior. Although previous works showed that the thermal properties of the neutron star core (for example, core neutrino emissivity) are more important [17, 18] for ignition conditions, a study on crust neutrino cooling may also be meaningful because crust neutrino cooling appears in a shallower column depth than core neutrino cooling.

Recently, Schatz et al. proposed that the Urca shell may exist in accreting neutron star crusts [19]. The Urca cooling mechanism [20], which proceeds back and forth between specific nuclei (or nucleons) and takes a great amount of energy away via neutrinos (and antineutrinos) [21], has been considered by Tsuruta and Cameron (TC70) in white dwarfs [22] and in Type-Ia supernovae [23, 24]. In TC70, the authors proposed that the thermal rounding of the Fermi surface or a vibrational oscillation will make phase space available for the Urca process to produce the Urca shell. For neutron stars, the thermal energy is pretty small compared with the Fermi energy, thus the standard neutron star model uses the zero-temperature approximation, in which case the parent nucleus $(Z, A)$ are transformed by capture of degenerate electrons into the daughter nucleus $(Z-1, A)$, while the daughter nucleus $(Z-1, A)$ cannot decay through $(Z-1, A) \rightarrow(Z, A)+e^{-}+\bar{v}_{e}$ because no phase space is available to re-emit the captured electrons. By considering the relatively high temperature $\left(T>10^{8} \mathrm{~K}\right)$ and possible low-lying excited states $E_{x} \lesssim k_{B} T$ [19], Schatz et al. showed that the crust Urca neutrino emissivity may be comparable with the crustal heating rate at the temperature in accreting neutron crusts (see Fig. 3 in their paper); thus the Urca pairs may have the potential to cool the outer crust, and make the surface layers thermally decouple from the deeper crust. 
If this is true, it will be a great challenge to current thermonuclear bursts models [25, 26].

Deibel et al. continued this work. They identified 85 odd$A$ isotopes that form Urca pairs in the neutron star ocean by combining with the crust Urca pairs identified by Schatz et al. and the Urca pairs abundances in X-ray burst and superburst ashes. They concluded that ocean Urca pairs will not have an impact on carbon ignition, while the strong crust Urca pairs may lower the ocean's steady-state temperature and increase carbon ignition depths [21].

We propose that, at such a large local mass accretion rate $\dot{m}=(0.1-0.3) \dot{m}_{E d d}$ in superbursting sources, the effect of compression on the Urca shell should be considered. Although it has been reported that superbursts may occur in neutron stars at near-local-Eddington mass accretion rate [2], a local accretion rate $\dot{m}=(0.1-0.3) \dot{m}_{E d d}$ is large enough to compress the Urca shell on a timescale shorter than the weakinteraction timescale. Thus, there may be a departures from chemical equilibrium for the the Urca processes. We strongly urge a study on deviated Urca processes. The reaction rate and neutrino emissivity should be calculated.

In this work, we present the "deviated shell" to distinguish it from the usual Urca shell in chemical equilibrium. In Sec. II, we present phase-space integrals of the reaction rate and neutrino emissivity. In Sec. III, we present the effect of accretion-driven compression on the Urca shell. Results and some discussions are presented in Sec. IV.

\section{PHASE SPACE INTEGRALS}

We consider the EC and $\beta^{-}$decay cycle between certain pairs of nuclei $(Z, A)$ and $(Z-1, A)$. $Z$ is the charge number and $A$ is the mass number.

$(Z, A)+e^{-} \rightarrow(Z-1, A)+v_{e}$,

$(Z-1, A) \rightarrow(Z, A)+e^{-}+\bar{v}_{e}$.

We assume the transition is only from ground state to ground state or from state $i$ of parent nuclei to state $f$ of daughter nuclei, $E_{i}$ and $E_{f}$ are the energies for state $i$ of nucleus $(Z, A)$ and state $f$ of nucleus $(Z-1, A)$, where $E_{i} \lesssim k_{B} T$, $E_{f} \lesssim k_{B} T$ according to Schatz et al., including the ground state. $k_{B}$ is the Boltzmann constant, $T$ is the temperature in units of K. We set $Q_{i f}$ as the energy difference between nuclei $(Z, A)$ and $(Z-1, A)$; thus, $Q_{i f}$ is always negative while $Q_{f i}$ is positive here, $Q_{i f}$ and $Q_{f i}$ in units of $\mathrm{MeV}$. This definition is slightly different from the energy difference between parent nuclei and daughter nuclei, but we think this helps avoid misunderstanding when simultaneously dealing with the EC and $\beta^{-}$decay processes. Besides, we set $M_{(Z, A)}$ and $M_{(Z-1, A)}$ to be the rest mass of the nuclei $(Z, A)$ and $(Z-1, A)$. We also define $W$ as the total energy of the relativistic electrons in units of electron rest mass $m_{e} c^{2}$. The threshold energy in units of electron rest mass can be expressed as

$q_{f i}=\frac{Q_{f i}}{m_{e} c^{2}}, Q_{f i}=M_{(Z-1, A)} c^{2}+E_{f}-M_{(Z, A)} c^{2}-E_{i}, q_{i f}=-q_{f i}$.

For a degenerate relativistic Fermi electron gas, the rate for- malism as derived by Fuller, Fowler and Newman ( FFN ) [27] applies. Thus the electron-capture rate (per nucleus per unit time) from state $i$ to state $f$ is

$$
\begin{aligned}
\lambda^{+} & =\frac{\ln 2}{f t} \Phi^{+}\left(q_{i f}\right), \\
\Phi^{+}\left(q_{i f}\right) & =\int_{w_{l}}^{\infty} F^{+}(Z, W) W P\left(W+q_{i f}\right)^{2} S(W) d W .
\end{aligned}
$$

For the $\beta^{-}$decay rate (per nucleus per unit time) from state $f$ to state $i$, we have

$$
\begin{aligned}
\lambda^{-} & =\frac{\ln 2}{f t} \Phi^{-}\left(q_{f i}\right), \\
\Phi^{-}\left(q_{f i}\right) & =\int_{1}^{w_{l}} F^{-}(Z, W) W P\left(q_{f i}-W\right)^{2}[1-S(W)] d W .(3)
\end{aligned}
$$

Similarly, the neutrino emissivity (energy per nucleus per unit time emitted via neutrinos) from state $i$ to state $f$ is

$$
\begin{aligned}
\xi^{+} & =\frac{\ln 2}{f t} m_{e} c^{2} \Psi^{+}\left(q_{i f}\right), \\
\Psi^{+}\left(q_{i f}\right) & =\int_{w_{l}}^{\infty} F^{+}(Z, W) W P\left(W+q_{i f}\right)^{3} S(W) d W
\end{aligned}
$$

The neutrino emissivity (energy per nucleus per unit time emitted via antineutrinos) from state $f$ to state $i$ is

$$
\begin{aligned}
\xi^{-} & =\frac{\ln 2}{f t} m_{e} c^{2} \Psi^{-}\left(q_{f i}\right), \\
\Psi^{-}\left(q_{f i}\right) & =\int_{1}^{w_{l}} F^{-}(Z, W) W P\left(q_{f i}-W\right)^{3}[1-S(W)] d W,(5)
\end{aligned}
$$

where $f t$ values measure the transition strength of EC and $\beta^{-}$ decay processes, $F^{ \pm}(Z, W)$ has been defined as the Coulomb correction factor, $w_{l}=1$ if $q_{i f}>-1$ or $w_{l}=\left|q_{i f}\right|$ if $q_{\text {if }}<-1$, and $S(W)$ is the Fermi-Dirac distribution function. Since electrons are relativistic and degenerate in accreting neutron stars crusts (except the outmost part), their large energies make $F^{ \pm}(Z, W)$ almost constant [28]. We take $\langle F\rangle^{ \pm} \simeq 2 \pi \alpha Z /\left|1-e^{\mp 2 \pi \alpha Z}\right|$, the average value of the factor $F^{ \pm}(Z, W)$ in our calculations, $\alpha$ is the fine structure constant. $\Phi^{ \pm}$and $\Psi^{ \pm}$can be expressed in what follows.

$$
\begin{aligned}
& \Phi^{+}=\left(\frac{k_{B} T}{m_{e} c^{2}}\right)^{5}\langle F\rangle^{+}\left[F_{4}(\eta)+2 \chi F_{3}(\eta)+\chi^{2} F_{2}(\eta)\right], \\
& \Phi^{-}=\left(\frac{k_{B} T}{m_{e} c^{2}}\right)^{5}\langle F\rangle^{-}\left[F_{4}(-\eta)-2 \chi F_{3}(-\eta)+\chi^{2} F_{2}(-\eta)\right], \\
& \Psi^{+}=\left(\frac{k_{B} T}{m_{e} c^{2}}\right)^{6}\langle F\rangle^{+}\left[F_{5}(\eta)+2 \chi F_{4}(\eta)+\chi^{2} F_{3}(\eta)\right], \\
& \Psi^{-}=\left(\frac{k_{B} T}{m_{e} c^{2}}\right)^{6}\langle F\rangle^{-}\left[F_{5}(-\eta)-2 \chi F_{4}(-\eta)+\chi^{2} F_{3}(-\eta)\right],
\end{aligned}
$$

where $\chi=Q_{f i} /\left(k_{B} T\right)$, and $\eta=\left(\mu_{e}+Q_{i f}\right) /\left(k_{B} T\right)=\delta \mu /\left(k_{B} T\right)$, keeping in mind that $\chi$ is positive. The function $F_{k}(\eta)$ is a 
relativistic Fermi integral of order $k$ defined as

$$
F_{k}(\eta)=\int_{0}^{\infty} \frac{y^{k}}{1+e^{y-\eta}} d y .
$$

\section{ACCRETION-DRIVEN COMPRESSION ON URCA SHELL}

Schatz et al. [19] proposed that the Urca shell spans a range of electron chemical potential $\left|Q_{i f}\right|-k_{B} T \lesssim \mu_{e} \lesssim\left|Q_{i f}\right|+k_{B} T$, the thickness of the Urca shell defined by the thermal fluctuations [13, 19] is $(\Delta R)_{\text {shell }} \approx\left|d R / d P_{e}\right|\left(d P_{e} / d \mu_{e}\right) \Delta \mu_{e} \approx$ $\left(d P_{e} / d \mu_{e}\right)\left(\Delta \mu_{e} / \rho g\right)$, where $R$ is the radius, $P_{e}$ is the degenerate pressure of electrons, $\rho$ is the mass density, and $g$ is the surface gravity. In this case, $\Delta \mu_{e}=k_{B} T,(\Delta R)_{\text {shell }} \approx Y_{e} k_{B} T /\left(m_{u} g\right)$, where $Y_{e} \approx Z / A$ is the electron fraction and $m_{u}$ is the atomic mass unit. EC and $\beta^{-}$decay proceed equally in the Urca shell, taking away a large amount of energy via neutrinos (and antineutrinos). For simplicity, we assume the Urca shell consists of only nuclei $(Z, A)$ and $(Z-1, A)$, with $n^{+}$being the number density of $(Z, A)$ and $n^{-}$being the number density of $(Z-1, A)$. In chemical equilibrium state, the steady state [22] gives

$$
n^{+} \lambda^{+}=n^{-} \lambda^{-}
$$

Combining Eqs. (2), (3), (6) and (7), it reads

$$
\begin{aligned}
& n^{+}\langle F\rangle^{+}\left[\chi^{2} F_{2}(0)+2 \chi F_{3}(0)+F_{4}(0)\right] \\
= & n^{-}\langle F\rangle^{-}\left[\chi^{2} F_{2}(0)-2 \chi F_{3}(0)+F_{4}(0)\right] .
\end{aligned}
$$

We define

$$
\kappa(\chi)=\frac{n^{+}\langle F\rangle^{+}}{n^{-}\langle F\rangle^{-}}=\frac{\chi^{2} F_{2}(0)-2 \chi F_{3}(0)+F_{4}(0)}{\chi^{2} F_{2}(0)+2 \chi F_{3}(0)+F_{4}(0)} .
$$

For large $\chi$ value, which means the energy threshold is large or the temperature is low, the $\chi^{2} F_{2}(0)$ term dominates, then $\kappa(\chi) \approx 1$. TC70 has used this approximation in white dwarfs with a temperature as low as $10^{4} \sim 10^{5} \mathrm{~K}$. But it is not appropriate for high temperature accreting neutron stars, for example, the Urca pair ${ }_{35}^{81} \mathrm{Br}-{ }_{34}^{81} \mathrm{Se}$ has a ground-state to groundstate threshold energy $\left|Q_{i f}\right|=1.59 \mathrm{MeV}$, at the temperature $T=4.5 \times 10^{8} \mathrm{~K}, \chi=41$, and $\kappa(\chi)=0.735$. Therefore, the parameter $\kappa(\chi)$ should be considered. Besides, the charge neutrality condition gives

$$
n_{e}=Z n^{+}+(Z-1) n^{-} \simeq Z\left(n^{+}+n^{-}\right),
$$

where $n_{e}$ is the electron number density. Combining Eqs. (11) and (14) one obtains

$$
n^{+} \approx \frac{n_{e}\langle F\rangle^{-} \kappa(\chi)}{Z\left[\langle F\rangle^{+}+\langle F\rangle^{-} \kappa(\chi)\right]}, n^{-} \approx \frac{n_{e}\langle F\rangle^{+}}{Z\left[\langle F\rangle^{+}+\langle F\rangle^{-} \kappa(\chi)\right]} .
$$

The net reaction rate is

$$
\begin{aligned}
\Gamma(T, \eta)= & n^{+} \lambda^{+}-n^{-} \lambda^{-} \\
= & \frac{C}{k_{B} T}\left[\chi^{2}\left(F_{2}(\eta)-\frac{F_{2}(-\eta)}{\kappa(\chi)}\right)\right. \\
& \left.+2 \chi\left(F_{3}(\eta)+\frac{F_{3}(-\eta)}{\kappa(\chi)}\right)+\left(F_{4}(\eta)-\frac{F_{4}(-\eta)}{\kappa(\chi)}\right)\right] \\
\approx & \frac{C}{k_{B} T} \chi^{2}\left[F_{2}(\eta)-\frac{F_{2}(-\eta)}{\kappa(\chi)}\right] .
\end{aligned}
$$

In the statistical equilibrium state, $\eta=0$ and $\Gamma(T, 0)=0$, the composition remains unchanged. The total neutrino energyloss rate is

$$
\begin{aligned}
\epsilon_{v}(T, \eta)= & n^{+} \xi^{+}+n^{-} \xi^{-} \\
= & C\left[\chi^{2}\left(F_{3}(\eta)+\frac{F_{3}(-\eta)}{\kappa(\chi)}\right)\right. \\
& \left.+2 \chi\left(F_{4}(\eta)-\frac{F_{4}(-\eta)}{\kappa(\chi)}\right)+\left(F_{5}(\eta)+\frac{F_{5}(-\eta)}{\kappa(\chi)}\right)\right] \\
\approx & C \chi^{2}\left[F_{3}(\eta)+\frac{F_{3}(-\eta)}{\kappa(\chi)}\right],
\end{aligned}
$$

where

$$
C=\frac{\ln 2}{f t} \frac{\left(k_{B} T\right)^{6}}{\left(m_{e} c^{2}\right)^{5}} \frac{n_{e}\langle F\rangle^{+}\langle F\rangle^{-} \kappa(\chi)}{Z\left[\langle F\rangle^{+}+\langle F\rangle^{-} \kappa(\chi)\right]} .
$$

The accretion-driven compression makes the Urca-shell scenario different. We consider only the effect of gravitational compression on the Urca shell under the weight of the newly accreted matter. On the one hand, compression makes the Urca shell denser and denser, resulting in an increase in electron number density. On the other hand, the Urca shell may deviate from chemical equilibrium as a response, resulting in a nonzero net reaction which tries to pull the electron number density back to the original level. Therefore, the Urca shell may be out of chemical equilibrium: we call this the deviated shell. The compression and the net reaction rate compete to determine the actual value $\delta \mu$, just like the case of gravitational contraction of npe (neutrons, protons and electrons) matter that Reisenegger [29] discussed in spin-down neutron stars. Or, in other words, the effect is the consequence of the competition between the timescale $\tau_{\text {com }}$ to increase $n_{e}$ by $\Delta n_{e}$ and the timescale $\tau_{\text {weak }}$ to consume electrons by $\Delta n_{e}$. The timescales $\tau_{\text {com }}$ and $\tau_{\text {weak }}$ are estimated in the following discussions.

We consider continual-accreting neutron stars, especially neutron stars accreting at high rates. For example, the superbursting sources accreting at $\dot{m}=(0.1-0.3) \dot{m}_{E d d}$. The weight of newly accreted matter pushes the matter beneath it to higher density and pressure; as a result, $n_{e}$ and $\mu_{e}$ keep rising. We can make a rough estimate of the increasing rate of $n_{e}: n_{e} \approx 4.3 \times 10^{30} \mu_{e}^{3} / \mathrm{cm}^{3}$, the pressure is dominated by relativistic degenerate electrons in the crust and has 
$P_{e} \approx 1.76 \times 10^{24} \mu_{\mathrm{e}}^{4} \mathrm{erg} / \mathrm{cm}^{3}, \mu_{e}$ in units of $\mathrm{MeV}$, thus

$$
\frac{d n_{e}}{d t} \approx \frac{d n_{e}}{d P_{e}} \frac{d P_{e}}{d t}=\frac{d n_{e}}{d P_{e}} \dot{m} g,
$$

where $g=G M / R^{2}$, and $M$ is the gravitational mass of the neutron star. For the representative values $R=10 \mathrm{~km}$ and $M=1.4 M_{\odot}$, where $M_{\odot}$ is the mass of the sun, $g=1.85 \times$ $10^{14} \mathrm{~cm} / \mathrm{s}^{2}$. We use $g=1.85 \times 10^{14} \mathrm{~cm} / \mathrm{s}^{2}$ for the Urca pairs throughout the crusts. Therefore,

$$
\frac{d n_{e}}{d t} \approx\left(3.43 \times 10^{25}\right) \frac{\dot{m} / \dot{m}_{E d d}}{\mu_{e}}\left(\mathrm{~cm}^{-3} \mathrm{~s}^{-1}\right) .
$$

Equation (19) shows that $d n_{e} / d t$ is proportional to the local accretion rate; thus, for superbursting sources, the high local accretion rates will increase $n_{e}$ dramatically. The timescale to increase electrons by $\Delta n_{e}$ is

$$
\tau_{c o m}=\frac{\Delta n_{e}}{d n_{e} / d t} \propto \frac{\left|Q_{i f}\right|}{\left(3.43 \times 10^{25}\right)\left(\dot{m} / \dot{m}_{E d d}\right)}(\mathrm{s}),
$$

while the timescale to consume electrons by $\Delta n_{e}$ is

$$
\begin{aligned}
\tau_{\text {weak }}(\eta)= & \frac{\Delta n_{e}}{\Gamma(T, \eta)} \propto \frac{Z f t}{\left(5.42 \times 10^{28}\right)\left|Q_{i f}\right|^{5} T_{9}^{3}\langle F\rangle^{*}} \\
& \times \frac{1}{F_{2}(\eta)-F_{2}(-\eta) / \kappa(\chi)}(\mathrm{s}),
\end{aligned}
$$

where $\langle F\rangle^{*}=\langle F\rangle^{+}\langle F\rangle^{-} \kappa(\chi) /\left[\langle F\rangle^{+}+\langle F\rangle^{-} \kappa(\chi)\right]$, and $T_{9}=$ $T /\left(10^{9} \mathrm{~K}\right)$. As long as the timescale satisfies the relation

$$
\tau_{\text {com }}<\tau_{\text {weak }}(\eta)
$$

the Urca shell must be a deviated one, we then obtain the upper limit of $\eta$ which identifies chemical departures from $\tau_{\text {com }}=\tau_{\text {weak }}(\eta)$, thus

$$
F_{2}(\eta)-\frac{F_{2}(-\eta)}{\kappa(\chi)}=\left(6.33 \times 10^{-4}\right) \frac{Z f t\left(\dot{m} / \dot{m}_{E d d}\right)}{\left|Q_{i f}\right|^{6} T_{9}^{3}\langle F\rangle^{*}} .
$$

We have used the Fermi integrals we derived in the appendix. Equation (23) shows that $\eta$ is more sensitive to temperature and energy thresholds than $f t$ values. Besides, experimentally measured $f t$ values have uncertainties. Hence, we fix $f t$ as a constant. The existence of a real solution to Eq. (23) determines whether a deviated shell is true. To make this specific, we discuss the effect of compression on Urca pairs in Table I of Deibel et al. [21], which are identified as the strongest. The majority of the corresponding $f t$ values fall in the range $5.2<\log f t<5.7$. For example, ${ }_{22}^{49} \mathrm{Ti}-{ }_{21}^{49} \mathrm{Sc}$ Urca pair has $\log f t=5.7,{ }_{25}^{55} \mathrm{Mn}-{ }_{24}^{55} \mathrm{Cr}$ has $\log f t=5.2$ and ${ }_{11}^{23} \mathrm{Na}-{ }_{10}^{23} \mathrm{Ne}$ has $\log f t=5.3$ [22]. For comparison, we take $\log f t=5.3$ and $T_{9}=0.45$ in the following calculations.

\begin{tabular}{|c|c|c|c|c|}
\hline Urca Pair, ${ }_{Z}^{A} \mathrm{X}$ & $\left|Q_{i f}\right|[\mathrm{MeV}]$ & $\eta^{a}\left[k_{B} T\right]$ & $\eta^{b}\left[k_{B} T\right]$ & $x$ \\
\hline${ }_{35}^{81} \mathrm{Br}-{ }_{34}^{81} \mathrm{Se}$ & 1.59 & 14.95 & 18.94 & 40.7 \\
\hline${ }_{22}^{49} \mathrm{Ti}-{ }_{21}^{49} \mathrm{Sc}$ & 2.00 & 6.96 & 8.98 & 51.2 \\
\hline${ }_{29}^{65} \mathrm{Cu}-{ }_{28}^{65} \mathrm{Ni}$ & 2.14 & 6.92 & 8.93 & 54.8 \\
\hline${ }_{25}^{55} \mathrm{Mn}-{ }_{24}^{55} \mathrm{Cr}$ & 2.60 & 3.87 & 5.21 & 66.6 \\
\hline${ }_{30}^{69} \mathrm{Zn}-{ }_{29}^{69} \mathrm{Cu}$ & 2.68 & 4.03 & 5.40 & 68.6 \\
\hline${ }_{26}^{57} \mathrm{Fe}^{*}-{ }_{25}^{57} \mathrm{Mn}$ & 2.70 & 3.57 & 4.84 & 69.1 \\
\hline${ }_{29}^{67} \mathrm{Cu}-{ }_{28}^{67} \mathrm{Ni}$ & 3.58 & 1.43 & 2.62 & 91.7 \\
\hline${ }_{28}^{63} \mathrm{Ni}^{*}-{ }_{27}^{63} \mathrm{Co}$ & 3.66 & 1.25 & 2.02 & 93.7 \\
\hline${ }_{12}^{25} \mathrm{Mg}-{ }_{11}^{25} \mathrm{Na}$ & 3.83 & 0.41 & 0.73 & 98.1 \\
\hline${ }_{34}^{81} \mathrm{Se}-{ }_{33}^{81} \mathrm{As}$ & 3.86 & 1.24 & 2.00 & 98.8 \\
\hline${ }_{31}^{73} \mathrm{Ga}-{ }_{30}^{73} \mathrm{Zn}$ & 4.11 & 0.80 & 1.39 & 105.2 \\
\hline${ }_{33}^{79} \mathrm{As}-{ }_{32}^{79} \mathrm{Ge}$ & 4.11 & 0.87 & 1.50 & 105.2 \\
\hline${ }_{11}^{23} \mathrm{Na}-{ }_{10}^{23} \mathrm{Ne}$ & 4.38 & 0.20 & 0.34 & 112.1 \\
\hline${ }_{42}^{101} \mathrm{Mo}^{*}-{ }_{41}^{101} \mathrm{Nb}$ & 4.63 & 0.69 & 1.21 & 118.5 \\
\hline${ }_{25}^{57} \mathrm{Mn}-{ }_{24}^{57} \mathrm{Cr}$ & 4.96 & 0.23 & 0.41 & 127.0 \\
\hline
\end{tabular}

Table I shows the upper limits of possible chemical departure values at the local accretion rate $\dot{m}=0.1 \dot{m}_{E d d}$ and $\dot{m}=0.2 \dot{m}_{E d d}$. In chemical departure cases, the net chemical energy release rate is $\Gamma(T, \eta) \delta \mu$, and the total neutrino energyloss rate is $\epsilon_{v}(T, \eta)=n^{+} \xi^{+}+n^{-} \xi^{-}$, thus the net cooling rate is
TABLE I: Ocean Urca pairs

${ }^{a}$ Calculated with $\log f t=5.3, T_{9}=0.45$ and $\dot{m}=0.1 \dot{m}_{E d d}$.

${ }^{b}$ Calculated with $\log f t=5.3, T_{9}=0.45$ and $\dot{m}=0.2 \dot{m}_{E d d}$.

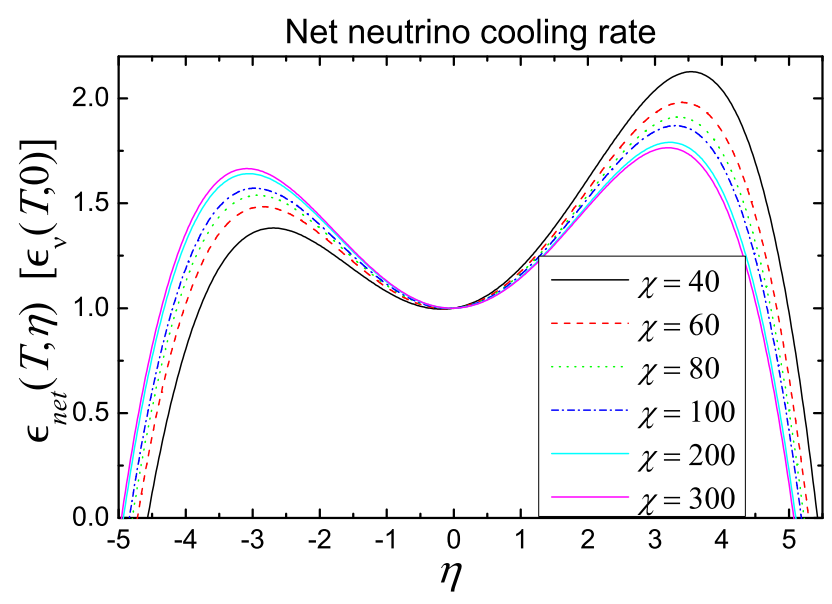

FIG. 1: Net cooling rate $\epsilon_{n e t}(T, \eta)$ in units of $\epsilon_{v}(T, 0)$. According to the $\chi$ values in Table I, we choose $\chi=40,60,80,100,200,300$ for illustrations. These lines have shown much in common, the dependence of net cooling rate on $\chi$ values (thus Urca pairs) is relatively weak, the net cooling rate will be enhanced if $0<\eta \lesssim 4.5$, when $\eta>5.5$, there will be no net cooling but net heating.

$\epsilon_{\text {net }}(T, \eta)=\epsilon_{\nu}(T, \eta)-\Gamma(T, \eta) \delta \mu$. The total cooling rate in the shell [21] is

$$
L(\eta)=4 \pi R^{2} \int_{\text {shell }} \epsilon_{\text {net }}(T, \eta) d z^{\prime},
$$

where $d z^{\prime}=\left(d P_{e} / d \mu_{e}\right)\left[d \mu_{e} /(\rho g)\right]$. We assume the deviated shell has the same composition and thickness with the Urca shell in chemical equilibrium. It is hard to tell the relation 
between the net chemical energy release rate $\Gamma(T, \eta) \delta \mu$ and the total neutrino energy-loss rate $\epsilon_{v}(T, \eta)$ according to Eqs. (16) and (17). When the approximation $\kappa(\chi) \approx 1$ is taken, Eqs. (16) and (17) can be expressed in units of $\epsilon_{\nu}(T, 0)$ analytically, that

$$
\Gamma(T, \eta) \delta \mu=\Gamma(T, \eta) \times k_{B} T \eta \simeq \epsilon_{v}(T, 0)\left(\frac{20 \eta^{2}}{7 \pi^{2}}+\frac{20 \eta^{4}}{7 \pi^{4}}\right),
$$

and

$$
\epsilon_{v}(T, \eta) \simeq \epsilon_{v}(T, 0)\left(1+\frac{30 \eta^{2}}{7 \pi^{2}}+\frac{15 \eta^{4}}{7 \pi^{4}}\right),
$$

where $\epsilon_{v}(T, 0)$ is the total neutrino energy-loss rate in chemical equilibrium. It is clear that the net chemical energy release rate increases faster than the total neutrino energy-loss rate, the heating and cooling compete, to a certain extent, net cooling will be replaced by net heating. The net cooling rate is presented in Fig. 1, which is plotted numerically according to Eqs. (16) and (17).

\section{RESULTS AND CONCLUSIONS}

We have investigated the effect of compression on Urca shells in fast accreting neutron stars. We find that, in neutron stars with local accretion rates $\dot{m}=(0.1-0.3) \dot{m}_{E d d}$, if the Urca shell exists in the ocean and crust of neutron stars, compression may make the Urca shell deviate from chemical equilibrium. The upper limits of chemical departure values for 15 strongest Urca pairs, which are calculated based on reasonable parameters in superbursting sources, are presented in Table I. Generally, the shallower the Urca shell exists, the bigger the upper limit of the chemical departure value is. At $\dot{m}=(0.1-0.3) \dot{m}_{E d d}$, effect of compression on Urca pairs with $\left|Q_{i f}\right|>4 \mathrm{MeV}$ will be very small, this means the Urca shell in the deeper crust (with higher $\left|Q_{i f}\right| \approx 10 \mathrm{MeV}$ ) may always be in chemical equilibrium even at the local Eddington accretion rate case.

As stated in the previous section, we assume the deviated shell has the same composition as that in chemical equilibrium. Under this assumption, we numerically calculated the net cooling rate. Figure 1 shows that the net cooling rate will be enhanced when $0<\eta \lesssim 4.5$, thus chemical departures may also make contributions to the total neutrino (and antineutrino) luminosity besides the Urca shell in chemical equilibrium. For the temperature (about $4 \times 10^{8} \mathrm{~K}$ ), the local accretion rate $\left[\dot{m}=(0.1-0.3) \dot{m}_{E d d}\right]$ and Urca pairs (in Table I) we are interested in, $\epsilon_{n e t}(T, \eta)$ will be enhanced by a factor of $1 \sim 2$. What is more, there will be no net cooling but net heating when $\eta \gtrsim 5.5$. Table I shows that the upper limits of ${ }_{35}^{81} \mathrm{Br}-{ }_{34}^{81} \mathrm{Se},{ }_{22}^{49} \mathrm{Ti}-{ }_{21}^{49} \mathrm{Sc}$ and ${ }_{29}^{65} \mathrm{Cu}-{ }_{28}^{65} \mathrm{Ni}$ are slightly bigger than 5.5 , which indicates that Urca pairs in shallow depth tend to result in net heating rather than cooling. Although the effect of heating can be pretty small, it is significant for accreting neutron stars because the crust Urca shell in chemical equilibrium proposed by Schatz et al. always acts as a parameterized cooling source. However, in consideration of the difference in accretion rate and threshold energy of Urca pairs, formation of a deviated shell makes the Urca-shell scenario differ from star to star and from Urca pair to Urca pair.

We conclude that, in accreting neutron stars, especially neutron stars accreting at a few tens of the local Eddington accretion rate, the Urca shell may deviate from chemical equilibrium, to what degree the Urca shell is deviated is determined by the properties of the nuclei, the temperature, and the actual local accretion rate. It is easier for Urca pairs in shallow depth to be deviated. The net cooling rate of these Urca pairs would be slightly enhanced if $0<\eta \lesssim 4.5$; however, net heating will appear if $\eta \gtrsim 5.5$. Only the strongest Urca pairs at deeper depth may always be in chemical equilibrium, indicating that these Urca pairs are most stable.

Deibel et al. have not considered Urca pairs with large $\log f t$ because neutrino emissivity of these pairs are much too small. We think, however, that Urca pairs with large $\log f t$ have more chances to be deviated from chemical equilibrium because the upper limits of $\eta$ can be larger. Does this mean that Urca shells are not stable structures in accreting neutron stars? We do not know yet; more constraints should be put on the actual $\eta$ (or $\delta \mu$ ). We hope this work may help to further understand some relevant astrophysical phenomena, such as Type-I x-ray bursts and superbursts. For more careful work, we think the effect of diffusion and convection [30-32] should also be discussed, but accretion-driven compression is certainly important.

\section{ACKNOWLEDGMENTS}

The authors would like to thank the anonymous referee very much for helpful comments, which have greatly improved our work. This work is supported by the National Natural Science Foundation of China (Grant No. 11178001) and CCNUQLPL Innovation Fund (Grant No. QLPL2015P01).

\section{APPENDIX}

The Fermi integrals may be calculated many times in the stellar evolution codes [27], thus simple and analytical expressions for them are needed. FFN had obtained good approximations for them by using the differential recursion relation $d F_{k}(\eta) / d \eta=k F_{k-1}(\eta)$ and $F_{0}(\eta)=\ln \left(1+\mathrm{e}^{\eta}\right)$. Based on these, they give

$$
\begin{aligned}
& F_{1}(\eta)+F_{1}(-\eta)=\frac{\eta^{2}}{2}+\frac{\pi^{2}}{6} \\
& F_{2}(\eta)-F_{2}(-\eta)=\frac{\eta^{3}}{3}+\frac{\pi^{2} \eta}{3} \\
& F_{3}(\eta)+F_{3}(-\eta)=\frac{\eta^{4}}{4}+\frac{\pi^{2} \eta^{2}}{2}+\frac{7 \pi^{4}}{60}, \\
& F_{4}(\eta)-F_{4}(-\eta)=\frac{\eta^{5}}{5}+\frac{2 \pi^{2} \eta^{3}}{3}+\frac{7 \pi^{4} \eta}{15} \\
& F_{5}(\eta)+F_{5}(-\eta)=\frac{\eta^{6}}{6}+\frac{5 \pi^{2} \eta^{4}}{6}+\frac{7 \pi^{4} \eta^{2}}{6}+\frac{31 \pi^{6}}{126}
\end{aligned}
$$


and

$$
\begin{aligned}
& F_{1}(\eta)= \begin{cases}e^{\eta}, & \eta \leqslant 0, \\
\frac{\eta^{2}}{2}+2-e^{-\eta}, & \eta>0,\end{cases} \\
& F_{2}(\eta)= \begin{cases}2 e^{\eta}, & \eta \leqslant 0, \\
\frac{\eta^{3}}{3}+\frac{\pi^{2} \eta}{3}+2 e^{-\eta}, & \eta>0,\end{cases} \\
& F_{3}(\eta)= \begin{cases}6 e^{\eta}, & \eta \leqslant 0, \\
\frac{\eta^{4}}{4}+\frac{\pi^{2} \eta^{2}}{2}+12-6 e^{-\eta}, & \eta>0,\end{cases} \\
& F_{4}(\eta)= \begin{cases}24 e^{\eta}, & \eta \leqslant 0, \\
\frac{\eta^{5}}{5}+\frac{2 \pi^{2} \eta^{3}}{3}+48 \eta+24 e^{-\eta}, & \eta>0,\end{cases} \\
& F_{5}(\eta)= \begin{cases}120 e^{\eta}, & \eta \leqslant 0, \\
\frac{\eta^{6}}{6}+\frac{5 \pi^{2} \eta^{4}}{6}+\frac{7 \pi^{4} \eta^{2}}{6}+240-120 e^{-\eta}, & \eta>0 .\end{cases}
\end{aligned}
$$

Their results are algebraically simple when $|\eta| \gg 0$, however, the largest error for $F_{k}(\eta)(\eta=1,2,3,4,5)$ in the range $|\eta| \leq 5$ can be up to $20 \%$, especially when $\eta \approx 0$. Here we propose an improvement of these Fermi integrals.

We do it by changing the integration limits. For odd $k$,

$F_{k}(\eta)+F_{k}(-\eta)=\int_{0}^{\infty} \frac{(y+\eta)^{k}+(y-\eta)^{k}}{1+e^{y}} d y-\int_{0}^{\eta}(y-\eta)^{k} d y$, and for even $k$,

$F_{k}(\eta)-F_{k}(-\eta)=\int_{0}^{\infty} \frac{(y+\eta)^{k}-(y-\eta)^{k}}{1+e^{y}} d y+\int_{0}^{\eta}(y-\eta)^{k} d y$.

According to Eqs. (37) and (38), we can reproduce the results in Eqs.(27)-(31). Besides, for odd $k$,

$$
\begin{aligned}
F_{k}(\eta)-F_{k}(-\eta)= & \int_{0}^{\infty} \frac{(y+\eta)^{k}-(y-\eta)^{k}}{1+e^{y}} d y \\
& -\int_{0}^{\eta} \frac{\left(e^{y}-1\right)(y-\eta)^{k}}{1+e^{y}} d y
\end{aligned}
$$

and for even $k$,

$$
\begin{aligned}
F_{k}(\eta)+F_{k}(-\eta)= & \int_{0}^{\infty} \frac{(y+\eta)^{k}-(y-\eta)^{k}}{1+e^{y}} d y \\
& +\int_{0}^{\eta} \frac{\left(e^{y}-1\right)(y-\eta)^{k}}{1+e^{y}} d y .
\end{aligned}
$$

When $|\eta|<2$, the factor $\left(e^{y}-1\right) /\left(e^{y}+1\right)$ can be expressed by Taylor expansion around $\eta=0$,

$$
\frac{e^{y}-1}{e^{y}+1} \approx \frac{y}{2}-\frac{y^{3}}{24}+\frac{y^{5}}{240} .
$$

Keeping the first two terms in Eq. (41) and Combining Eqs.
(27)-(31), we arrive at

$$
\begin{aligned}
F_{1}(\eta)= & \frac{\pi^{2}}{12}+(\ln 2) \eta+\frac{\eta^{2}}{4}+\frac{\eta^{3}}{24}-\frac{\eta^{5}}{960}, \\
F_{2}(\eta)= & \frac{3}{2} \zeta(3)+\frac{\pi^{2} \eta}{6}+(\ln 2) \eta^{2}+\frac{\eta^{3}}{6}+\frac{\eta^{4}}{48}-\frac{\eta^{6}}{2880}, \\
F_{3}(\eta)= & \frac{7 \pi^{4}}{120}+\frac{9}{2} \zeta(3) \eta+\frac{\pi^{2} \eta^{2}}{4}+(\ln 2) \eta^{3}+\frac{\eta^{4}}{8}+\frac{\eta^{5}}{80}-\frac{\eta^{7}}{6720}, \\
F_{4}(\eta)= & \frac{45 \zeta(5)}{2}+\frac{7 \pi^{4} \eta}{30}+9 \zeta(3) \eta^{2}+\frac{\pi^{2} \eta^{2}}{3}+(\ln 2) \eta^{4}+\frac{\eta^{5}}{10} \\
& +\frac{\eta^{6}}{120}-\frac{\eta^{8}}{13440}, \\
F_{5}(\eta)= & \frac{31 \pi^{4}}{256}+\frac{225}{2} \zeta(5) \eta+\frac{7 \pi^{4}}{12} \eta^{2}+15 \zeta(3) \eta^{3}+\frac{5 \pi^{2}}{12} \eta^{4} \\
& +(\ln 2) \eta^{5}+\frac{\eta^{6}}{12}+\frac{\eta^{7}}{168}-\frac{\eta^{9}}{24192},
\end{aligned}
$$

where $\zeta$ represent the Riemann zeta function, $\zeta(3)=1.20206$ and $\zeta(5)=1.03693$.

Finally, we get the complete expressions of Fermi integrals of order $k(k=1-5)$,

$$
F_{1}(\eta)= \begin{cases}e^{\eta}, & \eta \leqslant-2, \\ \frac{\pi^{2}}{12}+(\ln 2) \eta+\frac{\eta^{2}}{4}+\frac{\eta^{3}}{24}-\frac{\eta^{5}}{960}, & |\eta|<2, \\ \frac{\eta^{2}}{2}+2-e^{-\eta}, & \eta \geq 2\end{cases}
$$$$
F_{2}(\eta)= \begin{cases}2 e^{\eta}, & \eta \leqslant-2, \\ \frac{3}{2} \zeta(3)+\frac{\pi^{2} \eta}{6}+(\ln 2) \eta^{2}+\frac{\eta^{3}}{6}+\frac{\eta^{4}}{48} & \\ -\frac{\eta^{6}}{2880}, & |\eta|<2, \\ \frac{\eta^{3}}{3}+\frac{\pi^{2} \eta}{3}+2 e^{-\eta}, & \eta \geq 2,\end{cases}
$$

$F_{3}(\eta)=\left\{\begin{array}{lc}6 e^{\eta}, & \eta \leqslant-2 \\ \frac{7 \pi^{4}}{120}+\frac{9}{2} \zeta(3) \eta+\frac{\pi^{2} \eta^{2}}{4}+(\ln 2) \eta^{3} & \\ +\frac{\eta^{4}}{8}+\frac{\eta^{5}}{80}-\frac{\eta^{7}}{6720}, & |\eta|<2 \\ \frac{\eta^{4}}{4}+\frac{\pi^{2} \eta^{2}}{2}+\frac{7 \pi^{4}}{60}-6 e^{-\eta}, & \eta \geq 2\end{array}\right.$

$F_{4}(\eta)= \begin{cases}24 e^{\eta}, & \eta \leqslant-2, \\ \frac{45 \zeta(5)}{2}+\frac{7 \pi^{4} \eta}{30}+9 \zeta(3) \eta^{2}+\frac{\pi^{2} \eta^{2}}{3} & \\ +(\ln 2) \eta^{4}+\frac{\eta^{5}}{10}+\frac{\eta^{6}}{120}-\frac{\eta^{8}}{13440}, & |\eta|<2, \\ \frac{\eta^{5}}{5}+\frac{2 \pi^{2} \eta^{3}}{3}+\frac{7 \pi^{4} \eta}{15}+24 e^{-\eta}, & \eta \geq 2,\end{cases}$

$$
F_{5}(\eta)= \begin{cases}120 e^{\eta}, & \eta \leqslant-2 \\ \frac{31 \pi^{4}}{256}+\frac{225}{2} \zeta(5) \eta+\frac{7 \pi^{4}}{12} \eta^{2}+15 \zeta(3) \eta^{3} & \\ +\frac{5 \pi^{2}}{12} \eta^{4}+(\ln 2) \eta^{5}+\frac{\eta^{6}}{12}+\frac{\eta^{7}}{168}-\frac{\eta^{9}}{24192}, & |\eta|<2, \\ \frac{\eta^{6}}{6}+\frac{5 \pi^{2} \eta^{4}}{6}+\frac{7 \pi^{4} \eta^{2}}{6}+\frac{31 \pi^{6}}{126}-120 e^{-\eta} . & \eta \geq 2 .\end{cases}
$$

The above Fermi integral results for $F_{1}(\eta)$ through $F_{5}(\eta)$ are asymptotically exact for $|\eta| \geq 2$. The largest error appears around $\eta=-2$, about $3.94 \%$ for $F_{1}\left(\eta \rightarrow-2^{-}\right), 1.65 \%$ for $F_{2}(\eta=-2), 0.8 \%$ for $F_{3}(\eta=-2), 0.4 \%$ for $F_{4}(\eta=-2)$ 
and $0.2 \%$ for $F_{5}(\eta=-2)$. Thus we have got fine segmenting functions for Fermi integrals $F_{k}(\eta)$ of order $k=1,2,3,4,5$. It should be pointed out that these segmenting functions are not continuous at $\eta= \pm 2$. But when we are focusing on simplicity and accuracy, this improvement may be helpful.
[1] R. Cornelisse, J. Heise, E. kuulkers, F. Verbunt, and J. J. M. In't Zand, Astron. Astrophys. 357, L21 (2000).

[2] J. J. M. in't Zand, R. Cornelisse, and A. Cumming, Astron. Astrophys. 426, 257 (2004).

[3] T. E. Strohmayer and E. F. Brown, Astrophys. J. 566, 1045 (2002).

[4] R. Wijnands, Astrophys. J. 554, L59 (2001).

[5] P. Haensel and J. L. Zdunik, Astron. Astrophys. 227, 431 (1990).

[6] P. Haensel and J. L. Zdunik, Astron. Astrophys. 404, L33 (2003).

[7] S. Gupta, E. F. Brown, H. Schatz, P. Möller, and K-L. Kratz, Astrophys. J. 662, 1188 (2007).

[8] P. Haensel, J. L. Zdunik, Astron. Astrophys. 480, 459 (2008).

[9] S. E. Woosley and R. E. Taam, Nature (London) 263, 101 (1976).

[10] R. E. Taam and R. E. Picklum, Astrophys. J. 224, 210 (1978).

[11] E. F. Brown and L. Bildsten, Astrophys. J. 496, 915 (1998).

[12] A. Cumming and L. Bildsten, Astrophys. J. 559, L127 (2001).

[13] R. L. Cooper, A. W. Steiner, and E. F. Brown, Astrophys. J. 702, 660 (2009).

[14] A. Cumming, J. Macbeth, J. J. M. In't Zand, and D. Page, Astrophys. J. 646, 429 (2006).

[15] G. R. Caughlan and W. A. Fowler, At. Data Nucl. Data Tables 40, 283 (1988).

[16] K. Keek, A. Heger, and J. J. M. In’t Zand, Astrophys. J. 752, 150 (2012).
[17] E. F. Brown, Astrophys. J. 614, L57 (2004).

[18] R. L. Cooper and R. Narayan, Astrophys. J. 629, 422 (2005).

[19] H. Schatz, S. Gupta, P. Möller, M. Beard, E. F. Brown, A. T. Deibel, L. R. Gasques, W. R. Hix, L. Keek, R. Lau, A. W. Steiner and M. Wiescher, Nature (London) 505, 62 (2014).

[20] G. Gamow and M. Schoenberg, Phys. Rev. 59, 539 (1941).

[21] A. Deibel, Z. Meisel, H. Schatz, E. F. Brown and A. Cumming, arXiv, 1603. 01281v1.

[22] S. Tsuruta and A. G. W. Cameron, Astrophys. Space Sci. 7, 374 (1970).

[23] B. Paczynski, Astrophys. Lett. 11, 53 (1972).

[24] S. E. Woosley and T. A. Weaver, Ann. Rev. Astro. Astrophys. 24, 205 (1986).

[25] L. Keek and A. Heger, Astrophys. J. 743,189 (2011).

[26] S. E. Woosley, A. Heger, A. Cumming, R. D. Hoffman, J. Pruet, T. Rauscher, J. L. Fisker, H. Schatz, B. A. Brown and M. Wiescher, Astrophys. J. Suppl. Ser. 151, 75 (2004).

[27] G. M. Fuller, W. A. Fowler and M. J. Newman, Astrophys. J. 293, 1 (1985).

[28] G. Beaudet, E. E. Salpeter, and M. L. Silvestro, Astrophys. J. 174,79 (1972).

[29] A. Reisenegger, Astrophys. J. 442, 749 (1995).

[30] Z. Medin and A. Cumming, Astrophys. J. 730, 97 (2011).

[31] Z. Medin and A. Cumming, Astrophys. J. Lett. 783, L3 (2014).

[32] Z. Medin and A. Cumming, Astrophys. J. 702, 29 (2015). 